Check for updates

Cite this: J. Mater. Chem. C, 2020 8, 7034

Received 26th March 2020,

Accepted 26th April 2020

DOI: $10.1039 / \mathrm{d} 0 \mathrm{tc} 01541 \mathrm{e}$

rsc.li/materials-c

\section{Electric-controlled half-metallicity in magnetic van der Waals heterobilayer $\dagger$}

\author{
Cheng Tang, (D) ${ }^{a b}$ Lei Zhang, $^{\text {ab }}$ Stefano Sanvito ${ }^{c}$ and Aijun Du (D) $\star^{a b}$
}

\section{Introduction}

Half metallicity endows magnets with the ability to generate fully spin-polarized current, leading to a great potential of spintronics in highly efficient information storage and processing. ${ }^{1-3}$ This intriguing property has attracted considerable attention since being first realized in Mn-based Heusler alloys in $1983,{ }^{4}$ and it has been theoretically and/or experimentally discovered in several bulk materials, including ferromagnetic manganese perovskite, ${ }^{5}$ silicon-based heterostructure, ${ }^{6}$ metal-doped dilute magnetic semiconductor ${ }^{7}$ and transition metal oxide and fluoride ${ }^{8,9}$ In addition, half metallicity has also been theoretically demonstrated in a few two-dimensional (2D) structures, such as inorganic nanosheets containing transition metals, ${ }^{10-15}$ metal-organic frameworks ${ }^{16,17}$ and graphitic carbon nitride. ${ }^{18}$ In general, the successful discovery of $2 \mathrm{D}$ half-metals opens great opportunities for nanoscale spintronics. Unfortunately, in currently-known intrinsic half-metals, it is difficult to control the transition between the half-metallic and the semiconducting state, so that the fully spin-polarized current cannot be switched on and off at will. This is a major limitation undermining the design of controllable spintronic applications, including quantum computing and spin field effect transistor (FET).

\footnotetext{
${ }^{a}$ Centre for Materials Science, Queensland University of Technology, Gardens Point Campus, Brisbane, QLD 4000, Australia.E-mail: aijun.du@qut.edu.au

${ }^{b}$ School of Chemistry and Physics, Queensland University of Technology,

Gardens Point Campus, Brisbane, QLD 4000, Australia

${ }^{c}$ School of Physics and CRANN Institute, Trinity College, Dublin 2, Ireland

$\dagger$ Electronic supplementary information (ESI) available. See DOI: 10.1039/ dotc01541e
}

The realization of magnetic anisotropy promoted the discovery of 2D ferromagnetic materials. In 2017, $\mathrm{CrI}_{3}$ and $\mathrm{CrGeTe}_{3}{ }^{19,20}$ monolayers were successfully exfoliated from their bulk counterparts. Their stable structures and out-of-plane magnetization make them an interesting materials palette for magnetoelectronics, magneto-optics and ultrahigh-density memory devices. ${ }^{21-25}$ Their 2D nature enables the formation of complex heterostructures with attractive magnetoelectronic properties ${ }^{26-29}$ via van der Waals (vdW) interaction. Among them, magnetic bilayers are particularly interesting, since their interlayer magnetic coupling can be tuned to be either ferromagnetic (FM) or antiferromagnetic (AFM) by charge doping, element modification, external electric field and mechanical motion. ${ }^{11,27,30-34}$ In particular, electrically induced half-metallicity has been recently predicted in a few $2 \mathrm{D}$ systems with zero net magnetic moment, such as gapless graphene nanoribbons and small bandgap antiferromagnets. ${ }^{35,36}$ In these the electric field becomes a powerful tool for adjusting the spin-polarized electronic properties of 2D materials. To the best of our knowledge, notwithstanding massive efforts, ${ }^{27,37-39}$ controllable halfmetallicity has not been yet reported in $2 \mathrm{D}$ ferromagnetic layers both in experiments or theory. Thus the question is: can halfmetallicity be introduced and controlled in ferromagnetic bilayers?

Recently, magnetic heterostructures with intrinsic interlayer magnetic and electronic coupling have been widely studied as media for spin-Hall effect, multiferroicity, skyrmion generation and spin valves. ${ }^{40-44}$ The band structures of these vdW heterostructures can be tuned more efficiently than those of repeated multilayers, thus that magnetic heterobilayers appears as the 
ideal system for achieving electric-field-controlled magnetoelectronic properties. ${ }^{45,46}$ The $2 \mathrm{D}$ magnetic heterobilayers investigated to date are made by a combination of magnetic and nonmagnetic layers, ${ }^{46,47}$ a strategy that limits the possibility of manipulating magnetism and spin transport. With the goal of achieving controllable half-metallicity, here we propose a new-type of heterobilayer, composed of two different FM layers (FM-FM heterobilayer), possessing strong and asymmetric interlayer magnetoelectronic coupling.

In this work, we theoretically reveal that a half-metallic electronic structure can be induced and controlled in a new artificial $\mathrm{FM}$ semiconductor, $\mathrm{CrI}_{3}-\mathrm{GeGeTe}_{3}$ heterobilayers $(\mathrm{Cr}-\mathrm{H})$, by the application of a moderate electric field. $\mathrm{Cr}-\mathrm{H}$ can be experimentally fabricated, since both $\mathrm{CrI}_{3}$ and $\mathrm{CrGeTe}_{3}$ exist in their monolayer form and display well-matched lattice constants. By sliding the layers along the two high-symmetry directions, [100] and [110], we have discovered several energetically favourable stacking orders at global and local minima, and found that the interlayer magnetism can be either FM or ferrimagnetic (FiM) depending on the precise geometry of the bilayer. Most importantly, robust half-metallicity can be introduced by applying an electric field of $0.6 \mathrm{~V}^{-1}$ (in the direction from $\mathrm{CrGeTe}_{3}$ to $\mathrm{CrI}_{3}$ ). Moreover, the spin-polarized band crossing is also discovered in FM Cr-H under the continuously increased field. Herein, the mechanical analysis reveals that the external applied field offsets the electrostatic potential, leading to the electrically induced band alignment in heterobilayers. Our findings suggest that $\mathrm{Cr}-\mathrm{H}$ can be used for implementing efficient spin FETs in 2D, a device of great significance for both fundamental research and magnetoelectronic applications.

\section{Computational details}

Our calculations have been performed with density functional theory (DFT) ${ }^{48,49}$ as implemented in the Vienna ab initio Simulation Package (VASP), ${ }^{50,51}$ constructed over the projector-augmented wave (PAW) method. ${ }^{52}$ In order to determine the magnetic ground state, we have calculated the total energies of $\mathrm{Cr}-\mathrm{H}$ for different magnetic structures with the Perdew-Burke-Ernzerhof (PBE) parameterization of the generalized gradient approximation (GGA) ${ }^{53}$ to the exchange-correlation energy. Here, to describe the strong correlations between electrons in the $d$ shell, a Hubbard Coulomb on-site potential, $U$, of $3.0 \mathrm{eV}$ is set for $\mathrm{Cr}$, which is in consistent with previous calculations in $2 \mathrm{D} \mathrm{CrI}_{3} \cdot{ }^{30,31}$ Our results have also been extended to considering spin-orbit coupling (SOC) within GGA $+U .^{54}$ In order to obtain more accurate results and to eliminate the uncertainty associated with the empirical $U$ parameter, the state-of-the-art Heyd-Scuseria-Ernzerhof (HSE) hybrid functional ${ }^{55}$ has also been used. In all calculations, a vacuum gap larger than 20 Å was introduced to prevent the interactions between the outof-plane periodic replica of the unit cell. The plane-wave cut-off energy was set to $400 \mathrm{eV}$, while a Gamma-centred $k$-point meshes of $7 \times 7 \times 1$ was used to sample the first Brillouin zone. Dispersive vdW interactions were considered in our calculations through the empirical DFT-D3 corrections. The geometric structures were optimized without any constraint (unless noted otherwise) until the energy of each atoms converged to $10^{-6} \mathrm{eV}$ and the forces were less than $0.001 \mathrm{eV}^{-1}$.

The surface binding energies, $E_{\mathrm{b}}$, of $\mathrm{Cr}-\mathrm{H}$ for different stacking orders can be calculated as

$$
E_{\mathrm{b}}=E_{\mathrm{Cr}-\mathrm{H}}-E_{\mathrm{CrI}}-E_{\mathrm{CrGeTe}}
$$

where, the $E_{\mathrm{Cr}-\mathrm{H}}, E_{\mathrm{CrI}}$ and $E_{\mathrm{CrGeTe}}$ are the total energies of $2 \mathrm{D}$ $\mathrm{Cr}-\mathrm{H}, \mathrm{CrI}_{3}$ and $\mathrm{CrGeTe}_{3}$ nanosheets, respectively. From the DFT total energies of various magnetic configurations one can extract the spin-exchange coupling parameters, $J$, defined by the Heisenberg Hamiltonian,

$$
\begin{aligned}
H= & J_{\mathrm{CrI}} \sum_{\langle i j\rangle} \vec{S}_{i} \vec{S}_{j}+J_{\mathrm{CrGeTe}} \sum_{\langle k l\rangle} \vec{S}_{k} \vec{S}_{l}+J_{\text {inter }} \sum_{\langle\langle i k\rangle\rangle} \vec{S}_{i} \vec{S}_{k} \\
& +\sum_{i, k} K_{i, k}\left(\vec{S}_{i, k} \vec{A}_{i, k}\right)^{2}
\end{aligned}
$$

where, $J_{\mathrm{CrI}}$ and $J_{\mathrm{CrGeTe}}$ are the magnetic coupling constants of the nearest-neighboring $\mathrm{Cr}$ atoms within $\mathrm{CrI}_{3}$ and $\mathrm{CrGeTe}_{3}$ monolayers, respectively, and $J_{\text {inter }}$ represents the $\mathrm{Cr}-\mathrm{Cr}$ magnetic coupling across the interlayers ( $\vec{S}$ is the Cr magnetic moment). Here $K$ is the coefficient of magnetic anisotropy, and $\vec{A}$ represents the magnetocrystalline easy axis.

\section{Results and discussion}

The optimized in-plane lattice constants of $\mathrm{CrI}_{3}$ and $\mathrm{CrGeTe}_{3}$ monolayers are 6.92 and $6.90 \AA$, respectively. Therefore the mismatch in $\mathrm{Cr}-\mathrm{H}(6.91 \AA)$ is less than $1 \%$ within the primitive $(1 \times 1)$ bilayer cell. In Fig. $1 \mathrm{a}$ and $\mathrm{c}$, the top and side views of optimized $\mathrm{Cr}-\mathrm{H}$ for two different stacking orders $\left(\mathrm{AB}\right.$ and $\left.\mathrm{AB}^{\prime \prime}\right)$ are displayed. Compared to the $\mathrm{AB}$ stack, the $\mathrm{AB}^{\prime \prime}$ stack shows an interlayer twist between the top $\left(\mathrm{CrI}_{3}\right)$ and bottom $\left(\mathrm{CrGeTe}_{3}\right)$ layer with an angle of $60^{\circ}$. In order to investigate other possible stacking orders, we have displaced the two layers with respect to each other along the two high-symmetry directions, [100] and [110]. The position-dependent energies of the magnetic ground state is calculated as in previous work. ${ }^{30}$ The AB stack, presenting the lowest total energy and deepest surface binding energy (see Table 1), is at the global minimum among all the stacking orders investigated. However, several other structures with comparable energy are found at the local minima. These are labelled as $\mathrm{AA}, \mathrm{AB}^{\prime}, \mathrm{AC}_{1}{ }^{\prime}, \mathrm{AC}_{2}{ }^{\prime}, \mathrm{AD}_{1}{ }^{\prime}$ and $\mathrm{AD}_{2}{ }^{\prime}$ [see Fig. $1 \mathrm{~b}$ and $\mathrm{d}$ ], and they must be considered as metastable phases, kinetically available to be observed especially at a low temperature (note that the barriers for sliding are relatively high). It is worth mentioning that the $\mathrm{AC}_{2}{ }^{\prime}$ stack, obtained with a fractional lateral shift of the $\mathrm{CrI}_{3}$ unit cell of $[2 / 3,0]$ with respect to the starting $\mathrm{AB}^{\prime \prime}$ stack, is the most stable for an interlayer twisting angle of $60^{\circ}$.

We then calculate the energy difference between the FM and FiM spin alignment between the two monolayers (the difference in magnitude of the magnetic moments of $\mathrm{Cr}$ atoms in $\mathrm{CrI}_{3}$ and $\mathrm{CrGeTe}_{3}$ monolayers leads to net magnetic moment of $0.20 \mu_{\mathrm{B}}$ when setting the opposite interlayer spin configurations in $\mathrm{Cr}-\mathrm{H}$ ). We find that the interlayer magnetism of $\mathrm{Cr}-\mathrm{H}$ 

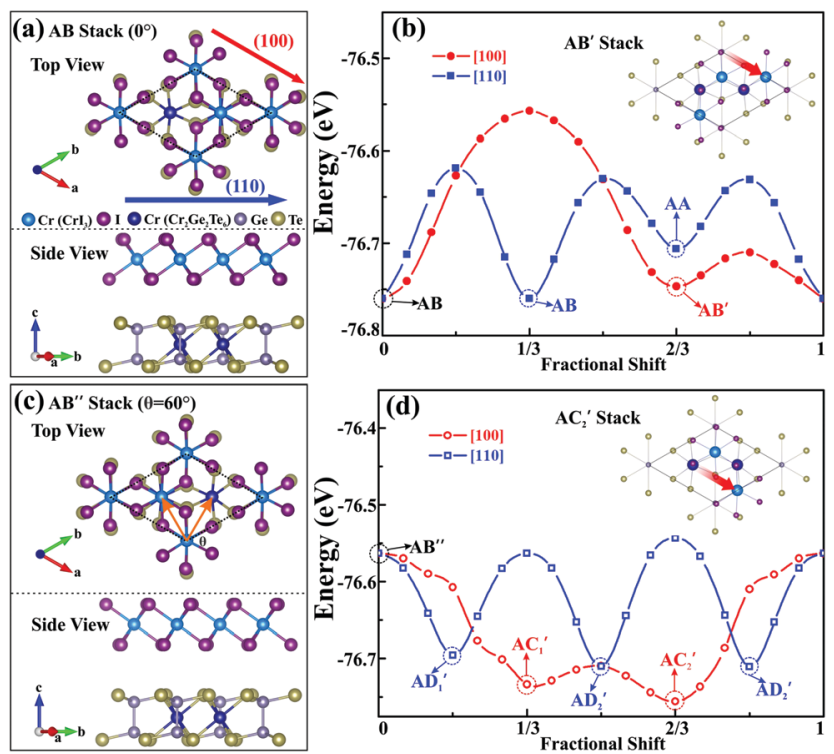

Fig. 1 Geometric structures of $\mathrm{Cr}-\mathrm{H}$ for the $A B$ [panel (a)] and $\mathrm{AB}^{\prime \prime}$ stacks [panel (c)]. Stacking energies as a function of the lateral shift, with respect to the (b) $A B$ and (d) $A B^{\prime \prime}$ stacks, respectively. Displacements along two high-symmetry directions, [100] (red lines) and [110] (blue lines), are explored. The stable and metastable stacking orders are marked in the figures. The top view of the $A B^{\prime}$ and $A C_{2}{ }^{\prime}$ stacks are inserted as insets in panels (b) and (d), with the red arrows representing the lateral shift between the layers.

Table 1 Magnetic ground states, total energies ( $E_{\text {tot, }}$ in eV), surface binding energies $\left(E_{\mathrm{b}}\right.$, in $\left.\mathrm{eV}\right)$, spin-exchange coupling parameters $\left(J\right.$, in $\left.\mathrm{meV} / \mu_{\mathrm{B}}{ }^{2}\right)$ and magnetic anisotropic energies (MAE $=E_{\text {in-plane }}-E_{\text {out-of-plane, }}$ positive values indicate out-of-plane magnetization, in $\mathrm{meV} / \mathrm{Cr}$ ) for different stacking orders in their magnetic ground states. The unit cell contains $4 \mathrm{Cr}$ atoms in total

\begin{tabular}{llllllll}
\hline & Ground state & $E_{\text {tot }}$ & $E_{\mathrm{b}}$ & $J_{\text {CrI }}$ & $J_{\text {CrGeTe }}$ & $J_{\text {inter }}$ & MAE \\
\hline $\mathrm{AB}$ & FM & -76.76 & -0.71 & -4.77 & -7.65 & -0.78 & 0.57 \\
$\mathrm{AA}$ & FM & -76.71 & -0.65 & -4.34 & -7.59 & -0.31 & 0.47 \\
$\mathrm{AB}{ }^{\prime}$ & FiM & -76.74 & -0.69 & -3.83 & -6.40 & 0.24 & 0.52 \\
$\mathrm{AC}^{\prime}{ }^{\prime}$ & FM & -76.74 & -0.68 & -4.44 & -6.85 & -0.12 & 0.59 \\
$\mathrm{AC}_{2}{ }^{\prime}$ & FiM & -76.75 & -0.70 & -3.76 & -6.45 & 0.35 & 0.48 \\
$\mathrm{AD}_{1}{ }^{\prime}$ & FiM & -76.70 & -0.64 & -4.08 & -6.63 & 0.29 & 0.50 \\
$\mathrm{AD}_{2}{ }^{\prime}$ & FiM & -76.71 & -0.65 & -3.76 & -6.35 & 0.21 & 0.49 \\
& & & & & & &
\end{tabular}

is strongly stacking-dependent (see Fig. S1 and S2 in the ESI $\dagger$ ), in line with what previously found for the $\mathrm{CrI}_{3}{ }^{30,32}$ and $\mathrm{CrS}_{2}{ }^{31}$ bilayers. The magnetic ground state of the $\mathrm{AB}, \mathrm{AA}$ and $\mathrm{AC}_{1}{ }^{\prime}$ stacks is $\mathrm{FM}$, whereas, that of $\mathrm{AB}^{\prime}, \mathrm{AC}_{2}{ }^{\prime}, \mathrm{AD}_{1}{ }^{\prime}$ and $\mathrm{AD}_{2}{ }^{\prime}$ is FiM (see Table 1 and Table S1, ESI $\dagger$ ). Fig. S3 (ESI $\dagger$ ) shows that the spin density, as expected, is mainly concentrated around the $\mathrm{Cr}$ atoms for both monolayers. An accurate analysis of the magnetic interaction based on the Heisenberg energy introduced before (see details in the ESI $\dagger$ ) reveals that the intralayer exchange-coupling parameters are about an order of magnitude larger than the interlayer ones (see Table 1), and close to those of $\mathrm{CrI}_{3}$ and $\mathrm{CrGeTe}_{3}$ monolayers. This suggests that $\mathrm{Cr}-\mathrm{H}$ may present a Curie (Néel) temperature similar to that of $2 \mathrm{D} \mathrm{CrI}_{3}$ and $\mathrm{CrGeTe}_{3}$. Notably, all of the stacking orders possess the out-of-plane magnetization (Table 1) with the values being closed to those of $\mathrm{CrI}_{3}$ and $\mathrm{CrGeTe}_{3}$ layers. ${ }^{56,57}$
After having investigated the ground-state magnetic order and strength of $\mathrm{Cr}-\mathrm{H}$, we now calculate their electronic properties with the $\mathrm{HSE}$ functional. Herein, the $\mathrm{AB}$ and $\mathrm{AC}_{2}{ }^{\prime}$ stacks are considered as two representative structures, not only because of their different magnetic ground state, but also because they are at the energy minimum for the interlayer twisting angles of $0^{\circ}$ and $60^{\circ}$, respectively. The $\mathrm{CrI}_{3}$ and $\mathrm{CrGeTe}_{3}$ monolayers are both FM semiconductors with bandgaps of 1.88 and $0.78 \mathrm{eV}$ (see Fig. S5, ESI $\dagger$ ), respectively. However, the bandgap of $\mathrm{Cr}-\mathrm{H}$ reduces to around $0.55 \mathrm{eV}$ regardless of the precise stacking order (Fig. S6, ESI $\dagger$ ), due to the intrinsic band alignment. Interestingly, the valence band maximum (VBM) and conduction band minimum (CBM) of $\mathrm{FM} \mathrm{Cr-H}$ are in the same spin channel, whereas those of the FiM states straddle different spin channels. In order to demonstrate this point the orbitalresolved density of states near the Fermi level of $\mathrm{Cr}-\mathrm{H}$ is plotted in Fig. S7 (ESI $\dagger$ ). We can see that the CBM is composed of $\mathrm{Cr} 3 \mathrm{~d}$ and I $5 p$ orbitals from the $\mathrm{CrI}_{3}$ layer, while the VBM is mainly contributed by Te $5 p$ orbitals originating from $\mathrm{CrGeTe}_{3}$.

Next we investigate the effects of the external electric field on the magnetic and electronic properties of $\mathrm{Cr}-\mathrm{H}$. As shown in Fig. 2, the energy difference (blue lines) between the FM and FiM configurations is significantly reduced with increasing the intensity of the applied field for both the $\mathrm{AB}$ and $\mathrm{AC}_{2}{ }^{\prime}$ stacks. The exchange parameters are remarkably decreased with the increased external field (Fig. S8, ESI $\dagger$ ), indicating a stronger interlayer FM coupling. For the AB stack the magnetic coupling between the layers remains FM for all fields, while for the $\mathrm{AC}_{2}{ }^{\prime}$ one there is a FiM-FM transition at an applied field of $-0.8 \mathrm{~V} \AA^{-1}$. Interestingly, the magnetic moment of the $\mathrm{Cr}$ ions in $\mathrm{CrGeTe}_{3}$ depends little on the electric field (Fig. S9, ESI $\dagger$ ), while that of $\mathrm{CrI}_{3}$ increases significantly for positive fields (the direction of the field is from $\mathrm{CrGeTe}_{3}$ to $\mathrm{CrI}_{3}$, see Fig. 2a), owing to an electrically induced charge distribution. At $1.0 \mathrm{~V} \AA^{-1}$ the net magnetization of the $\mathrm{CrI}_{3}$ layer increases from $3.47 \mu_{\mathrm{B}}$ at zero field to $3.54 \mu_{\mathrm{B}}$, indicating an increment of $1.69 \times 10^{13} \mathrm{~cm}^{-2}$ carriers in this layer. ${ }^{36}$

Most importantly, robust half-metallicity is introduced at a positive electric field. As we can see in Fig. 2, for the $\mathrm{AB}$ and
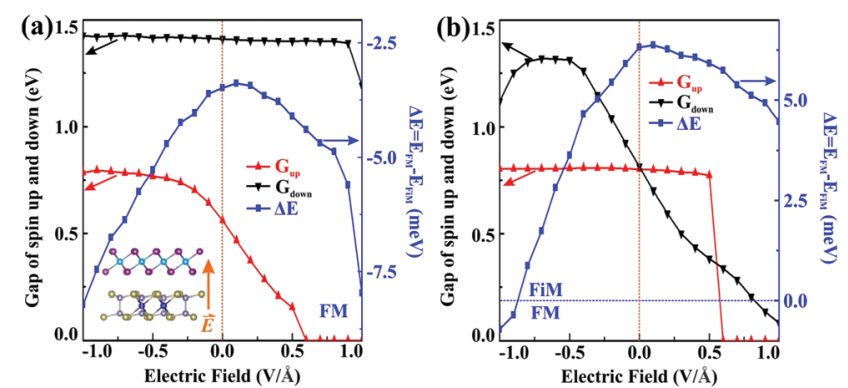

Fig. 2 Electric field dependent band gaps of spin up (red) and down (black) states and total energy difference (blue) between the FM and FiM configurations for (a) the $\mathrm{AB}$ and (b) $\mathrm{AC}_{2}$ ' stacks. $G_{\text {up }}$ and $G_{\text {down }}$ indicate the band gaps in the spin up and spin down channel, respectively. As a matter of convention the electric field is positive when pointing from $\mathrm{CrGeTe}_{3}$ to $\mathrm{Crl}_{3}$. 
$\mathrm{AC}_{2}{ }^{\prime}$ stacks, the spin up channel becomes metallic with the band gap dropping to zero at a field of $0.6 \mathrm{~V} \AA^{-1}$, while the spin down channel remains semiconducting with a band gap of 1.40 and $0.34 \mathrm{eV}$, respectively for $\mathrm{AB}$ and $\mathrm{AC}_{2}{ }^{\prime}$. For comparison, $\mathrm{a}$ similar electric-field-induced transition between the semiconducting and half-metallic states cannot be achieved in $\mathrm{CrI}_{3}$ and $\mathrm{CrGeTe}_{3}$ homo-bilayers, where the two spin channels simultaneously turn metallic after reaching a critical field $\left(1.2 \mathrm{~V}^{-1}\right.$ for $\mathrm{CrI}_{3}$ and $1.0 \mathrm{~V}^{-1}$ for $\mathrm{CrGeTe}_{3}$, see details in the ESI $\dagger$ ). Therefore, the magnetic heterobilayer with proper band alignment is an effective heterojunction to achieve the electrically controllable half-metallicity, ascribing by the asymmetry of the band structure.

The evolution of the band structure as a function of the electric field (for fields of $-0.6,0,0.6,0.8$ and $1.0 \mathrm{~V}^{-1}$ ) is shown in Fig. S11 (ESI $\dagger$ ) and schematically in Fig. 3 for both the $\mathrm{AB}$ and $\mathrm{AC}_{2}{ }^{\prime}$ stacks. In the absence of an external field, the VBMs of two spin channels of the $\mathrm{AB}\left(\mathrm{AC}_{2}{ }^{\prime}\right)$ stack are both made of $\mathrm{Te} \mathrm{p}_{x}$ and $\mathrm{p}_{y}$ orbitals, while the CBMs are respectively contributed by $\mathrm{Cr} \mathrm{t}_{2 \mathrm{~g}}$ orbitals from the $\mathrm{CrI}_{3}\left(\mathrm{CrGeTe}_{3}\right)$ and $\mathrm{CrGeTe}_{3}\left(\mathrm{CrI}_{3}\right)$ layers. The difference in orbital composition of the CBM owes to the different magnetic order of the two stacks, obtained by reversing the local moment of the $\mathrm{CrI}_{3}$ layer. As one can observe in Fig. 3, when the electric field gets larger than the critical field of $0.6 \mathrm{~V} \AA^{-1}$ only the spin up channel crosses the Fermi level, while the spin down band structure remains that of a semiconductor with a band gap of $1.40 \mathrm{eV}(0.20 \mathrm{eV})$ for the $\mathrm{AB}$ $\left(\mathrm{AC}_{2}{ }^{\prime}\right)$ stack. The Fermi surface is thus characterized by spin up Te $\mathrm{p}_{x}$ and $\mathrm{p}_{y}$ orbitals, suggesting a half-metallic conductivity. Patently, at the field of $0.8 \mathrm{~V}^{-1}$, Te p and $\mathrm{Cr} \mathrm{t}_{2 \mathrm{~g}}$ (from $\mathrm{CrI}_{3}$ ) orbitals are crossing in the spin up channel of the $A B$ stack, which can be confirmed by the decomposed charge density of bands near Fermi level. For the band of the AB stack, the charge density in spin up channel at $0.8 \mathrm{~V}^{-1}$ distributes in two layers (Fig. 3c), whereas that at $0 \mathrm{~V}^{-1}$ are contributed by only one layer (Fig. S12a, ESI $\dagger$ ). However, the $\mathrm{AC}_{2}{ }^{\prime}$ stack possesses similar charge density distribution at 0 and $0.8 \mathrm{~V}^{-1}$.

Fig. 4 schematically illustrates the band alignment of the FM and FiM state of the heterojunction. The work function of monolayer $\mathrm{CrI}_{3}$ is $5.95 \mathrm{eV}$, much larger than that of $\mathrm{CrGeTe}_{3}$ $(4.89 \mathrm{eV})$. Based on such difference in workfunction and in the band gap of the constituent monolayers, the intrinsic band alignment of the FM (FiM) state of $\mathrm{Cr}-\mathrm{H}$ is type II for spin up (down), and type I for the other spin channel. This also makes the minimum band gap, $G_{\text {up-down }}$, of FiM Cr-H straddling two spin channels. The external electric field offsets the electrostatic potential and shifts the band positons of heterobilayers. As shown in Fig. 4b and c, both of the CBM of $\mathrm{CrI}_{3}$ and VBM of $\mathrm{CrGeTe}_{3}$ are approaching to the Fermi level under the positive electric field, while moving inversely under the negative field. In addition, the electrically induced charge density, defined as $\Delta \rho=\rho(\vec{E})-\rho(0)$, distinctly shows the opposite charge distribution in two outermost atomic layers (Fig. S14, ESI $\dagger$ ).

The ability to engineer its band structure opens up the possibility to use $\mathrm{CrI}_{3}-\mathrm{CrGeTe}_{3}$ heterobilayers to generate spin-polarized current pulses. Fig. S15a (ESI $\dagger$ ) gives an example of a spin FET. The voltage applied through the top and back gates $^{36}$ provides the input. As discussed above, one of the spin channels of $\mathrm{Cr}-\mathrm{H}$ can be tuned from semiconducting to metallic, while the other spin channel remains semiconducting at the critical perpendicular electric field. Therefore, in such

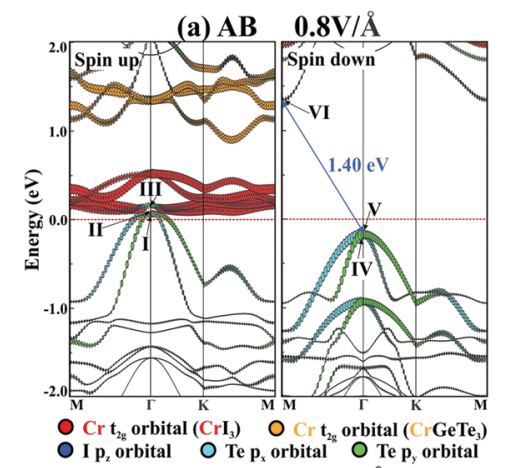

(c) $\mathbf{A B}$
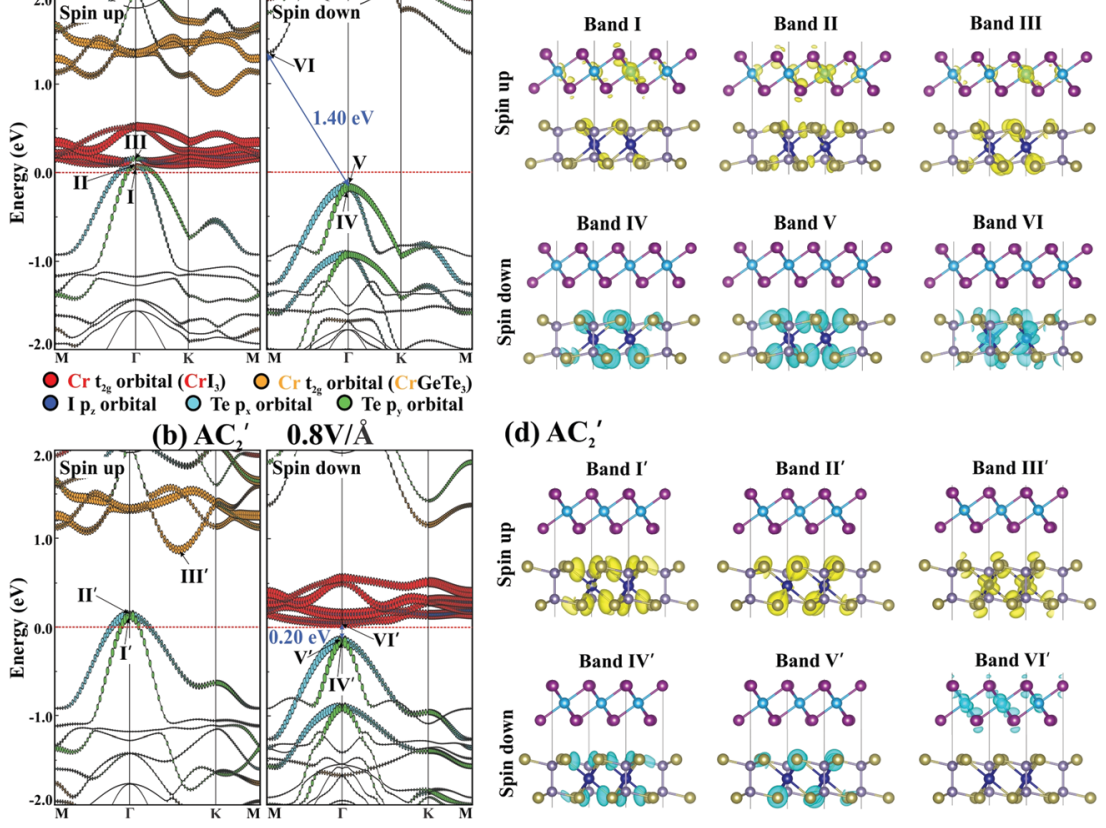

(d) $\mathrm{AC}_{2}^{\prime}$
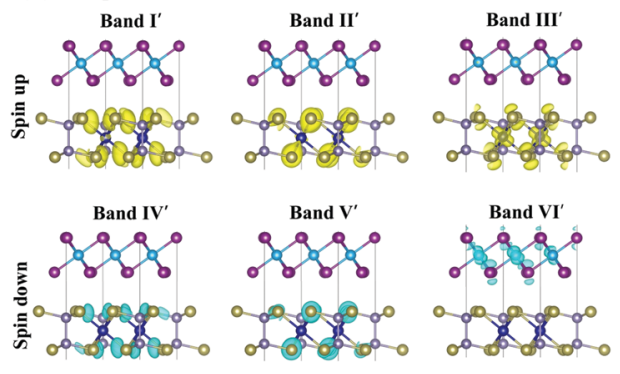

Fig. 3 Orbital-resolved band structures of the (a) $A B$ and (c) $A C_{2}{ }^{\prime}$ stacks under an external electric field of $0.8 \vee \AA^{-1}$. Decomposed charge densities corresponding to the marked position of the bands for the (b) $\mathrm{AB}$ and (d) $\mathrm{AC}_{2}{ }^{\prime}$ stacking orders. 
(a)

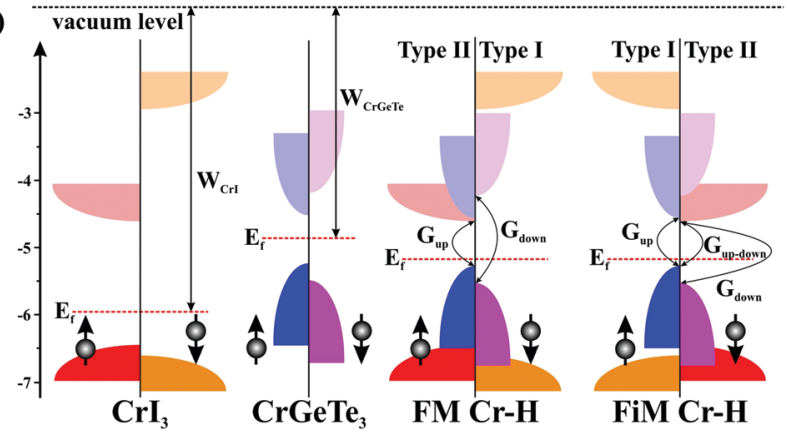

(b) AB stack (FM)



(c) $\mathrm{AC}_{2}^{\prime}$ stack (FiM)

$$
-0.6 \mathrm{~V} / \AA ̊
$$
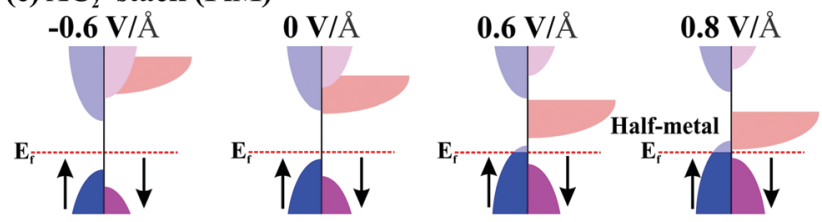

Fig. 4 Schematic band diagram for the (a) FM and FiM order and both (b) the $A B$ and (c) $\mathrm{AC}_{2}{ }^{\prime}$ stacks under different external electric field. Only the bands near Fermi level are considered in (b) and (c). Red and Orange areas represent the valence band of $\mathrm{Crl}_{3}$ spin up and down channel, while Blue and pink areas are $\mathrm{CrGeTe}_{3}$ spin up and down channels, respectively. The corresponding light colors represent the conduction bands.

spin FET, when the amplitude of the alternating current (AC) voltage exceeds the critical voltage (Fig. S15b, ESI $\dagger$ ), a spinpolarized pulse current will be obtained between the source and drain. In addition, the period of these generated current can be easily modified by controlling the input AC voltage.

\section{Conclusions}

In summary, we have explored theoretically the magnetoelectronic properties of experimentally feasible $\mathrm{CrI}_{3}-\mathrm{CrGeTe}_{3}$ heterobilayers with electric-controlled half-metallicity. Seven energetically favourable stacking orders of $\mathrm{Cr}-\mathrm{H}$ are discovered at the global and local minima. Their intralayer magnetic coupling is FM, while the interlayer one can be manipulated between FM and FiM by altering the stacking geometry (FM for $\mathrm{AB}, \mathrm{AA}$ and $\mathrm{AC}_{1}{ }^{\prime}$ stacks, $\mathrm{FiM}$ for $\mathrm{AB}^{\prime}, \mathrm{AC}_{2}{ }^{\prime}, \mathrm{AD}_{1}{ }^{\prime}$ and $\mathrm{AD}_{2}{ }^{\prime}$ stacks). Notably, in electric field pointing from $\mathrm{CrGeTe}_{3}$ to $\mathrm{CrI}_{3}$ of $0.6 \mathrm{~V}^{-1}$, one spin channel makes the transition from semiconducting to metallic, while the other remains semiconducting. This leads to the transport of $100 \%$ spinpolarized current. Furthermore, the spin-polarized interlayer band crossing near Fermi level is introduced in the type II spin channel of $\mathrm{FM} \mathrm{Cr}-\mathrm{H}$ by the continuously increased field. Herein, the important role of the applied field is to offset the electrostatic potential in heterobilayer, resulting in the electriccontrolled band alignment. Based on our findings, we propose a spin FET device able to generate $100 \%$ spin-polarized pulses. The mechanism for electric-field-induced half-metallicity in $\mathrm{Cr}-\mathrm{H}$ can be extended to other magnetic heterobilayers, suggesting the great potential of this materials class for controllable magnetoelectronic and spintronic applications.

\section{Conflicts of interest}

There are no conflicts to declare.

\section{Acknowledgements}

A. D. acknowledges the financial support by Australian Research Council under Discovery Project (DP170103598) and computer resources provided by high-performance computer time from computing facility at the Queensland University of Technology, NCI National Facility, and The Pawsey Supercomputing Centre through the National Computational Merit Allocation Scheme supported by the Australian Government and the Government of Western Australia. SS thanks the Irish Research Council Advanced Laureate Award (IRCLA/2019/127) for financial support.

\section{References}

1 S. Wolf, D. Awschalom, R. Buhrman, J. Daughton, S. Von Molnar, M. Roukes, A. Y. Chtchelkanova and D. Treger, Spintronics: a spin-based electronics vision for the future, Science, 2001, 294(5546), 1488-1495.

2 C. Chappert, A. Fert and F. N. Van Dau, The emergence of spin electronics in data storage, Nat. Mater., 2007, 6(11), 813-823.

$3 \mathrm{X}$. Hu, Half-metallic antiferromagnet as a prospective material for spintronics, Adv. Mater., 2012, 24(2), 294-298.

4 R. De Groot, F. Mueller, P. Van Engen and K. Buschow, New class of materials: half-metallic ferromagnets, Phys. Rev. Lett., 1983, 50(25), 2024.

5 J.-H. Park, E. Vescovo, H.-J. Kim, C. Kwon, R. Ramesh and T. Venkatesan, Direct evidence for a half-metallic ferromagnet, Nature, 1998, 392(6678), 794.

$6 \mathrm{H}$. Wu, P. Kratzer and M. Scheffler, Density-functional theory study of half-metallic heterostructures: interstitial Mn in Si, Phys. Rev. Lett., 2007, 98(11), 117202.

7 J. E. Medvedeva, A. J. Freeman, X. Cui, C. Stampfl and N. Newman, Half-metallicity and efficient spin injection in AlN/GaN: Cr (0001) heterostructure, Phys. Rev. Lett., 2005, 94(14), 146602.

8 Y. Jiao, F. Ma, C. Zhang, J. Bell, S. Sanvito and A. Du, Firstprinciples prediction of spin-polarized multiple Dirac rings in manganese fluoride, Phys. Rev. Lett., 2017, 119(1), 016403.

9 R. Keizer, S. Goennenwein, T. Klapwijk, G. Miao, G. Xiao and A. Gupta, A spin triplet supercurrent through the halfmetallic ferromagnet $\mathrm{CrO}_{2}$, Nature, 2006, 439(7078), 825.

10 B. Wang, Y. Zhang, L. Ma, Q. Wu, Y. Guo, X. Zhang and J. Wang, $\mathrm{MnX}$ (X = P, As) monolayers: a new type of two- 
dimensional intrinsic room temperature ferromagnetic half-metallic material with large magnetic anisotropy, Nanoscale, 2019, 11(10), 4204-4209.

$11 \mathrm{X} . \mathrm{Li}, \mathrm{X}$. Wu and J. Yang, Half-metallicity in $\mathrm{MnPSe}_{3}$ exfoliated nanosheet with carrier doping, J. Am. Chem. Soc., 2014, 136(31), 11065-11069.

12 T. Cao, Z. Li and S. G. Louie, Tunable magnetism and halfmetallicity in hole-doped monolayer GaSe, Phys. Rev. Lett., 2015, 114(23), 236602.

13 Y. Li, D. West, H. Huang, J. Li, S. Zhang and W. Duan, Theory of the Dirac half metal and quantum anomalous Hall effect in Mn-intercalated epitaxial graphene, Phys. Rev. B: Condens. Matter Mater. Phys., 2015, 92(20), 201403.

14 J. He, S. Ma, P. Lyu and P. Nachtigall, Unusual Dirac halfmetallicity with intrinsic ferromagnetism in vanadium trihalide monolayers, J. Mater. Chem. C, 2016, 4(13), 2518-2526.

15 Q. Wu, Y. Zhang, Q. Zhou, J. Wang and X. C. Zeng, Transition-Metal Dihydride Monolayers: A New Family of Two-Dimensional Ferromagnetic Materials with Intrinsic Room-Temperature Half-Metallicity, J. Phys. Chem. Lett., 2018, 9(15), 4260-4266.

16 C. Tang, C. Zhang, Z. Jiang, K. Ostrikov and A. Du, Theoretical discovery of Dirac half metal in experimentally synthesized two dimensional metal semiquinoid frameworks, J. Mater. Chem. C, 2019, 7(19), 5792-5796.

17 Y. Ma, Y. Dai, X. Li, Q. Sun and B. Huang, Prediction of twodimensional materials with half-metallic Dirac cones: $\mathrm{Ni}_{2} \mathrm{C}_{18} \mathrm{H}_{12}$ and $\mathrm{Co}_{2} \mathrm{C}_{18} \mathrm{H}_{12}$, Carbon, 2014, 73, 382-388.

18 A. Du, S. Sanvito and S. C. Smith, First-principles prediction of metal-free magnetism and intrinsic half-metallicity in graphitic carbon nitride, Phys. Rev. Lett., 2012, 108(19), 197207.

19 B. Huang, G. Clark, E. Navarro-Moratalla, D. R. Klein, R. Cheng, K. L. Seyler, D. Zhong, E. Schmidgall, M. A. McGuire, D. H. Cobden, W. Yao, D. Xiao, P. Jarillo-Herrero and $\mathrm{X}$. Xiaodong, Layer-dependent ferromagnetism in a van der Waals crystal down to the monolayer limit, Nature, 2017, 546(7657), 270.

20 C. Gong, L. Li, Z. Li, H. Ji, A. Stern, Y. Xia, T. Cao, W. Bao, C. Wang and Y. Wang, Discovery of intrinsic ferromagnetism in two-dimensional van der Waals crystals, Nature, 2017, 546(7657), 265.

21 Y. J. Deng, Y. J. Yu, Y. C. Song, J. Z. Zhang, N. Z. Wang, Z. Y. Sun, Y. F. Yi, Y. Z. Wu, S. W. Wu, J. Y. Zhu, J. Wang, $X$. H. Chen and Y. B. Zhang, Gate-tunable room-temperature ferromagnetism in two-dimensional $\mathrm{Fe}_{3} \mathrm{GeTe}_{2}$, Nature, 2018, 563(7729), 94.

22 Z. Y. Fei, B. Huang, P. Malinowski, W. B. Wang, T. C. Song, J. Sanchez, W. Yao, D. Xiao, X. Y. Zhu, A. F. May, W. D. Wu, D. H. Cobden, J. H. Chu and X. D. Xu, Two-dimensional itinerant ferromagnetism in atomically thin $\mathrm{Fe}_{3} \mathrm{GeTe}_{2}$, Nat. Mater., 2018, 17(9), 778.

23 J. Xiao, H. Zhu, Y. Wang, W. Feng, Y. Hu, A. Dasgupta, Y. Han, Y. Wang, D. A. Muller and L. W. Martin, Intrinsic two-dimensional ferroelectricity with dipole locking, Phys. Rev. Lett., 2018, 120(22), 227601.
24 C. Zheng, L. Yu, L. Zhu, J. L. Collins, D. Kim, Y. Lou, C. Xu, M. Li, Z. Wei and Y. Zhang, Room temperature in-plane ferroelectricity in van der Waals $\mathrm{In}_{2} \mathrm{Se}_{3}, S c i$. Adv., 2018, 4(7), eaar7720.

25 M. Bonilla, S. Kolekar, Y. Ma, H. C. Diaz, V. Kalappattil, R. Das, T. Eggers, H. R. Gutierrez, M.-H. Phan and M. Batzill, Strong room-temperature ferromagnetism in $\mathrm{VSe}_{2}$ monolayers on van der Waals substrates, Nat. Nanotechnol., 2018, 13(4), 289.

26 B. Huang, G. Clark, D. R. Klein, D. MacNeill, E. NavarroMoratalla, K. L. Seyler, N. Wilson, M. A. McGuire, D. H. Cobden, D. Xiao, W. Yao, P. Jarillo-Herrero and $\mathrm{X}$. Xiaodong, Electrical control of 2D magnetism in bilayer $\mathrm{CrI}_{3}$, Nat. Nanotechnol., 2018, 13(7), 544.

27 E. S. Morell, A. Leon, R. H. Miwa and P. Vargas, Control of magnetism in bilayer $\mathrm{CrI}_{3}$ by an external electric field, $2 D$ Mater., 2019, 6(2), 25020.

28 S. Jiang, J. Shan and K. F. Mak, Electric-field switching of twodimensional van der Waals magnets, Nat. Mater., 2018, 1.

29 T. Song, X. Cai, M. W.-Y. Tu, X. Zhang, B. Huang, N. P. Wilson, K. L. Seyler, L. Zhu, T. Taniguchi and K. Watanabe, Giant tunneling magnetoresistance in spin-filter van der Waals heterostructures, Science, 2018, 360(6394), 1214-1218.

30 N. Sivadas, S. Okamoto, X. Xu, C. J. Fennie and D. Xiao, Stacking-dependent magnetism in bilayer $\mathrm{CrI}_{3}$, Nano Lett., 2018, 18(12), 7658-7664.

31 C. Wang, X. Zhou, Y. Pan, J. Qiao, X. Kong, C.-C. Kaun and $\mathrm{W}$. Ji, Layer and doping tunable ferromagnetic order in twodimensional $\mathrm{CrS}_{2}$ layers, Phys. Rev. B, 2018, 97(24), 245409.

32 P. Jiang, C. Wang, D. Chen, Z. Zhong, Z. Yuan, Z.-Y. Lu and W. Ji, Stacking tunable interlayer magnetism in bilayer CrI 3, Phys. Rev. B, 2019, 99(14), 144401.

33 E.-J. Kan, Z. Li, J. Yang and J. Hou, Half-metallicity in edgemodified zigzag graphene nanoribbons, J. Am. Chem. Soc., 2008, 130(13), 4224-4225.

$34 \mathrm{X} . \mathrm{Li}, \mathrm{X}$. Wu and J. Yang, Room-temperature half-metallicity in $\mathrm{La}(\mathrm{Mn}, \mathrm{Zn}) \mathrm{AsO}$ alloy via element substitutions, J. Am. Chem. Soc., 2014, 136(15), 5664-5669.

35 Y.-W. Son, M. L. Cohen and S. G. Louie, Half-metallic graphene nanoribbons, Nature, 2006, 444(7117), 347.

36 S.-J. Gong, C. Gong, Y.-Y. Sun, W.-Y. Tong, C.-G. Duan, J.-H. Chu and $\mathrm{X}$. Zhang, Electrically induced 2D half-metallic antiferromagnets and spin field effect transistors, Proc. Natl. Acad. Sci. U. S. A., 2018, 115(34), 8511-8516.

37 B. Huang, G. Clark, D. R. Klein, D. MacNeill, E. NavarroMoratalla, K. L. Seyler, N. Wilson, M. A. McGuire, D. H. Cobden and D. Xiao, Electrical control of 2D magnetism in bilayer $\mathrm{CrI}_{3}$, Nat. Nanotechnol., 2018, 13(7), 544.

38 H. Ohno, D. Chiba, F. Matsukura, T. Omiya, E. Abe, T. Dietl, Y. Ohno and K. Ohtani, Electric-field control of ferromagnetism, Nature, 2000, 408(6815), 944.

39 L.-T. Chang, C.-Y. Wang, J. Tang, T. Nie, W. Jiang, C.-P. Chu, S. Arafin, L. He, M. Afsal and L.-J. Chen, Electric-field control of ferromagnetism in Mn-doped $\mathrm{ZnO}$ nanowires, Nano Lett., 2014, 14(4), 1823-1829.

40 Y. Lv, J. Kally, D. Zhang, J. S. Lee, M. Jamali, N. Samarth and J.-P. Wang, Unidirectional spin-Hall and Rashba-Edelstein 
magnetoresistance in topological insulator-ferromagnet layer heterostructures, Nat. Commun., 2018, 9(1), 111.

41 I. Lemesh, K. Litzius, M. Böttcher, P. Bassirian, N. Kerber, D. Heinze, J. Zázvorka, F. Büttner, L. Caretta and M. Mann, Current-Induced Skyrmion Generation through Morphological Thermal Transitions in Chiral Ferromagnetic Heterostructures, Adv. Mater., 2018, 30(49), 1805461.

42 S. V. Eremeev, M. M. Otrokov and E. V. Chulkov, New universal type of interface in the magnetic insulator/topological insulator heterostructures, Nano Lett., 2018, 18(10), 6521-6529.

43 C. Gong, E. M. Kim, Y. Wang, G. Lee and X. Zhang, Multiferroicity in atomic van der Waals heterostructures, Nat. Commun., 2019, 10(1), 2657.

44 C. Cardoso, D. Soriano, N. García-Martínez and J. FernándezRossier, van der Waals spin valves, Phys. Rev. Lett., 2018, 121(6), 067701.

45 A. K. Geim and I. V. Grigorieva, van der Waals heterostructures, Nature, 2013, 499(7459), 419-425.

46 Q. Tong, H. Yu, Q. Zhu, Y. Wang, X. Xu and W. Yao, Topological mosaics in moiré superlattices of van der Waals heterobilayers, Nat. Phys., 2017, 13(4), 356.

47 N. Lu, H. Guo, L. Li, J. Dai, L. Wang, W.-N. Mei, X. Wu and X. C. Zeng, $\mathrm{MoS}_{2} / \mathrm{MX}_{2}$ heterobilayers: bandgap engineering via tensile strain or external electrical field, Nanoscale, 2014, 6(5), 2879-2886.

48 L. Sham and M. Schlüter, Density-functional theory of the energy gap, Phys. Rev. Lett., 1983, 51(20), 1888.
49 E. Gross and W. Kohn, Local density-functional theory of frequency-dependent linear response, Phys. Rev. Lett., 1985, 55(26), 2850.

50 G. Kresse and J. Furthmüller, Efficient iterative schemes for ab initio total-energy calculations using a plane-wave basis set, Phys. Rev. B: Condens. Matter Mater. Phys., 1996, 54(16), 11169.

51 G. Kresse and J. Furthmüller, Efficiency of $a b$ initio total energy calculations for metals and semiconductors using a plane-wave basis set, Comput. Mater. Sci., 1996, 6(1), 15-50.

52 G. Kresse and D. Joubert, From ultrasoft pseudopotentials to the projector augmented-wave method, Phys. Rev. B: Condens. Matter Mater. Phys., 1999, 59(3), 1758.

53 J. P. Perdew, K. Burke and M. Ernzerhof, Generalized gradient approximation made simple, Phys. Rev. Lett., 1996, 77(18), 3865.

54 S. Dudarev, G. Botton, S. Savrasov, C. Humphreys and A. Sutton, Electron-energy-loss spectra and the structural stability of nickel oxide: An LSDA $+U$ study, Phys. Rev. B: Condens. Matter Mater. Phys., 1998, 57(3), 1505.

55 J. Heyd, G. E. Scuseria and M. Ernzerhof, Hybrid functionals based on a screened Coulomb potential, J. Chem. Phys., 2003, 118(18), 8207-8215.

56 J. L. Lado and J. Fernández-Rossier, On the origin of magnetic anisotropy in two dimensional $\mathrm{CrI}_{3}, 2 D$ Mater., 2017, 4(3), 035002.

57 J. Zeisner, A. Alfonsov, S. Selter, S. Aswartham, M. Ghimire, M. Richter, J. van den Brink, B. Büchner and V. Kataev, Magnetic anisotropy and spin-polarized two-dimensional electron gas in the van der Waals ferromagnet $\mathrm{Cr}_{2} \mathrm{Ge}_{2} \mathrm{Te}_{6}$, Phys. Rev. B, 2019, 99(16), 165109. 